\title{
BMJ Open Mesh fixation methods in open inguinal hernia repair: a protocol for network meta-analysis and trial sequential analysis of randomised controlled trials
}

\author{
Long Ge, ${ }^{1,2,3}$ Jin-hui Tian, ${ }^{2,3}$ Lun Li, ${ }^{4}$ Quan Wang, ${ }^{5}$ Ke-hu Yang ${ }^{2,3}$
}

To cite: Ge L, Tian J-hui, Li L, et al. Mesh fixation methods in open inguinal hernia repair: a protocol for network meta-analysis and trial sequential analysis of randomised controlled trials BMJ Open 2015;5:e009369. doi:10.1136/bmjopen-2015009369

- Prepublication history for this paper is available online. To view these files please visit the journal online (http://dx.doi.org/10.1136/ bmjopen-2015-009369).

LG and J-hT are co-first authors.

Received 11 July 2015 Revised 25 October 2015 Accepted 27 October 2015

CrossMark

For numbered affiliations see end of article.

Correspondence to Professor Ke-hu Yang; kehuyangebm2006@126.com

\section{ABSTRACT}

Introduction: Randomised clinical trials (RCTs) have been used to compare and evaluate different types of mesh fixation usually employed to repair open inguinal hernia. However, there is no consensus among surgeons on the best type of mesh fixation method to obtain optimal results. The choice often depends on surgeons' personal preference. This study aims to compare different types of mesh fixation methods to repair open inguinal hernias and their role in the incidences of chronic groin pain, risk of hernia recurrence, complications, operative time, length of hospital stay and postoperative pain, using Bayesian network meta-analysis and trial sequential analysis of RCTs.

Methods and analysis: A systematic search will be performed using PubMed, EMBASE, the Cochrane Central Register of Controlled Trials (CENTRAL), Chinese Biomedical Literature Database (CBM) and Chinese Journal Full-text Database, to include RCTs of different mesh fixation methods (or fixation vs no fixation) during open inguinal hernia repair. The risk of bias in included RCTs will be evaluated according to the Cochrane Handbook V.5.1.0. Standard pairwise meta-analysis, trial sequential analysis and Bayesian network meta-analysis will be performed to compare the efficacy of different mesh fixation methods.

Ethics and dissemination: Ethical approval and patient consent are not required since this study is a meta-analysis based on published studies. The results of this network meta-analysis and trial sequential analysis will be submitted to a peer-reviewed journal for publication.

Protocol registration number: PROSPERO CRD42015023758.

\section{INTRODUCTION}

The inguinal hernia, a common health issue, is a protrusion of abdominal contents into the inguinal canal through an abdominal wall defect, and its repair represents one of the most common surgical procedures. The lifetime rate of inguinal hernia is $2 \%$ in

\section{Strengths and limitations of this study}

- To the best of our knowledge, this is the first network meta-analysis and trial sequential analysis protocol comparing different types of mesh fixation methods to repair open inguinal hernias.

- The results of this systematic review will help clinicians and patients to select appropriate mesh fixation methods.

- Our results will be limited by both the quantity and quality of the trials available for review.

females and $25 \%$ in males. ${ }^{12}$ However, the risk of inguinal hernia increases with age, from $0.25 \%$ at 18 years of age to $4.2 \%$ at 75 80 years of age. ${ }^{3}$ Surgical repair of inguinal hernias is the most common general surgical procedure in the world. ${ }^{4}$ It is already well known that the surgical procedure of inguinal hernia repair is generally represented by one of the following three procedures: open repair with a mesh, open repair without the use of a mesh implant (ie, sutured) and laparoscopic repair with a mesh. ${ }^{5}$ One of the main problems associated with open mesh repair is chronic groin pain, ${ }^{6}$ which can be reduced using laparoscopic inguinal hernia repair. ${ }^{7}$ However, open mesh repair still plays an important role in the repair of inguinal hernias because it is generally less expensive and easy to perform, can be performed under local anaesthesia, and it is the method chosen when laparoscopic repair has failed. ${ }^{8}$

The current surgical options for mesh fixation include, but are not limited to, sutures, tacks or staples, self-fixing meshes and fibrin or other glues. ${ }^{9}$ However, there is no consensus among doctors on the best surgical technique. The choice of options often depends on surgeons' personal preference. ${ }^{9}$ Two meta-analyses compared glue fixation to suture fixation, and concluded that glue 
fixation was superior to suture fixation, especially regarding the reduction of chronic groin pain and its short operative time. ${ }^{6}{ }^{10}$ Five meta-analyses comparing self-gripping mesh with sutured mesh suggested that selfgripping mesh was associated with shorter operative time compared to sutured mesh. ${ }^{11-15}$ All of these meta-analyses conducted only pairwise meta-analysis to compare efficacy of different types of mesh fixation methods. Moreover, they lack information required for size sample calculation (sample size included in pooling outcomes). To evaluate the effects of different types of mesh fixation methods in open inguinal hernia repair, highly compelling and persuasive evidence is required to draw a firm conclusion.

Network meta-analysis has become increasingly popular to evaluate healthcare interventions, since it allows for estimation of the relative effectiveness among all interventions and rank ordering of the interventions even if head-to-head comparisons are lacking. ${ }^{16}$ Trial sequential analysis (TSA) is a tool for quantifying the statistical reliability of the data in a cumulative meta-analysis, ${ }^{17}{ }^{18}$ controlling $\alpha$ and $\beta$ values for sparse data and repetitive testing on accumulation data. ${ }^{19} 20$

This study is a comprehensive network meta-analysis and TSA on different types of mesh fixation available for open inguinal hernia repair.

\section{OBJECTIVE}

The objectives of this study are to compare the role of different types of mesh fixation in the incidences of chronic groin pain, risk of hernia recurrence, complications, operative time, length of hospital stay and postoperative pain for open inguinal hernia repair using Bayesian network meta-analysis and TSA of randomised clinical trials (RCTs).

\section{METHODS AND ANALYSIS}

\section{Design}

Bayesian network meta-analysis and TSA will be carried out in this study.

\section{Registration}

We registered on the international prospective register of systematic reviews (PROSPERO) to publish our study protocol. The protocol of network meta-analysis is performed according to the preferred reporting items for systematic review and meta-analysis protocol (PRISMA-P) recommendation, and the PRISMA extension statement for reporting of systematic reviews incorporating network meta-analyses of healthcare interventions. ${ }^{21} 22$

\section{Information source}

A systematic search will be performed using PubMed, EMBASE, the Cochrane Central Register of Controlled Trials (CENTRAL), Chinese Biomedical Literature Database (CBM) and Chinese Journal Full-text Database. The search strategy will be developed by LG and JHT, who have over 10 years of experience as information specialists. The references of included articles and reviews will be tracked to identify other relevant studies. We will not contact authors for detailed information of primary studies.

\section{Search strategy}

Search terms will be: inguinal hernia, groin hernia, inguinal hernioplasty, mesh, random* and others. Full details of the search strategy regarding PubMed, EMBASE and CENTRAL are:

PubMed: (('Inguinal hernia[Title/Abstract] OR 'groin hernia[Title/Abstract] OR 'inguinal hernioplasty[Title/ Abstract] OR 'Hernia, Inguinal[Mesh]) AND (mesh [Title/Abstract])) AND (Random*[All Fields] OR 'randomized controlled trial*[All Fields] OR 'randomized trial*[All Fields] OR Randomized Controlled Trial[ptyp] OR 'Randomized Controlled Trials as Topic[Mesh])

EMBASE: ('inguinal hernia'/exp/mj OR 'inguinal hernia' OR 'groin hernia'/exp/mj OR 'groin hernia' OR 'inguinal hernioplasty') AND (mesh) AND (random* OR[controlled clinical trial]/lim OR(randomized controlled trial]/lim) NOT [MEDLINE]/lim

CENTRAL: \#1 'Inguinal hernia' or 'groin hernia' or 'inguinal hernioplasty':ti, ab, kw (Word variations have been searched)

\#2 MeSH descriptor:[Hernia, Inguinal] explode all trees \#3 \#1 OR \#2

\#4 mesh:ti, ab, kw (Word variations have been searched) \#5 random*

\#6 \#3 AND \#4 AND \#5.

\section{Eligibility criteria}

Type of patients: adults (aged 18 years or older) with inguinal hernia, who scheduled for open inguinal hernia repair. The open mesh repair includes all kinds of techniques, such as Stoppa, Lichtenstein and mesh plug. Patients will be excluded if the hernia is inoperable with open inguinal hernia repair, or if the hernia repair technique changed to another one (eg, laparoscopic access methods).

Type of designs: truly random or quasi-random controlled trials; systematic reviews or meta-analyses will also be included to track their references.

Type of interventions: different mesh fixation methods (or fixation vs no fixation) in open inguinal hernia repair, including, but not limited to, sutures, tacks or staples, self-fixing meshes and fibrin or other glues.

Type of outcomes: the primary outcomes are incidence of chronic groin pain and risk of hernia recurrence. The secondary outcomes include complications, operative time, length of hospital stay and postoperative pain. The end points are defined in table 1 .

Other criteria: we will include RCTs reported in the English and Chinese languages. There will be no limitations on year of publication, publication status, duration of study follow-up or period of study conduct. 
Table 1 Data extraction items

\begin{tabular}{|c|c|}
\hline Category & Description \\
\hline \multicolumn{2}{|l|}{ Patient characteristics } \\
\hline Median age & Median age and range of included patients \\
\hline Type of inguinal hernia & $\begin{array}{l}\text { Unilateral or bilateral inguinal hernia; medial, lateral, femoral (and combinations); size of hernia } \\
\text { defect/opening }\end{array}$ \\
\hline Details of intervention & Details on different mesh fixation methods \\
\hline Size of mesh & Size of mesh used \\
\hline Type of mesh & Material, pore size, weight \\
\hline Number of tacks & Details on number and types of tacks \\
\hline Mesh fixation methods & Details on other types of fixation \\
\hline Follow-up & Period of follow-up and lost to follow-up \\
\hline \multicolumn{2}{|l|}{ Study characteristics } \\
\hline First authors & Name of the first author \\
\hline Year of publication & PubTime of included trials \\
\hline Study arms & Details on the intervention and control group \\
\hline Sample size & Sample size of included trials \\
\hline Type of design & Type of design of included trials \\
\hline \multicolumn{2}{|l|}{ Outcomes } \\
\hline \multirow[t]{2}{*}{ Chronic groin pain } & Groin pain persisting at least 3 months after the index operation \\
\hline & Visual analogue scale (VAS) $\geq 40 \mathrm{~mm}$ if scoring system was utilised \\
\hline Recurrence & Clinical or radiologic recurrence of inguinal hernia \\
\hline Postoperative pain & VAS immediately after and during 1 week of the operation \\
\hline Complications & Any complications requiring further procedures in the theatre during the same surgical admission \\
\hline Operative time & Time from skin incision to skin closure \\
\hline Length of hospital stay & Time from the index operation to discharge \\
\hline
\end{tabular}

\section{Study records}

Literature search records will be imported into ENDNOTE X6 literature management software, while a standard data abstraction form will be created using Microsoft Excel 2013 (Microsoft Corp, Redmond, WA, http://www.microsoft.com) to collect data of interest. A pilot test will be performed for literature selection and data extraction, and a 'cheat sheet' with detailed definitions and examples will be developed to ensure high inter-rater reliability among the reviewers.

Two independent reviewers will examine the title and abstract of studies found in the search, to identify related studies according to eligibility criteria. Thus, fulltext versions of all potentially relevant studies will be obtained. Excluded trials and the reasons for their exclusion will be listed and examined by a third reviewer.

To extract the data, a rigorous process will be used. First, a draft data extraction form will be conducted. Subsequently, a random sample of five included RCTs will be pilot tested, and the $\mathrm{k}$ statistic will be calculated. The form will be revised, as necessary, to confirm the final data extraction form. Finally, two reviewers will independently extract the data of interest, and conflicts will be resolved by a third reviewer.

\section{Data items}

We will extract all data of interest from each included RCT, including patient characteristics, study characteristics and outcomes. Data extraction item details can be found in table 1. We will consider the following factors as effect modifiers: median age, intervention, size and type of mesh, number and type of fixation, follow-up, and sample size.

\section{Risk of bias of individual studies}

The risk of bias of included RCTs will be evaluated according to the Cochrane Handbook V.5.1.0, ${ }^{23}$ including method of random sequence generation (selection bias), allocation concealment (selection bias), blinding (performance bias and detection bias), incomplete outcome data (detection bias), selective reporting (detection bias) and other bias. We will evaluate methodological quality as low, high or unclear risk of bias. The risk of bias assessment will be completed by two independent reviewers, and conflicts will be resolved by a third reviewer.

\section{Dealing with missing data}

We will not contact authors to obtain missing information of primary studies. If binary outcomes are missing, we will perform an available-case analysis, but we will assess the impact of 'best-best', 'best-worst', 'worst-best' and 'worst-worst' scenario analyses. ${ }^{24}$ Regarding the continuous outcomes, we will impute the mean from median and SD for SE, interquartile range, or $\mathrm{p}$ values, according to the Cochrane Handbook for Systematic Reviews of Interventions. If such studies are included, we will perform a sensitivity analysis to assess their impact. 


\section{Standard pairwise meta-analysis}

We will perform pairwise meta-analysis, using STATA V.12.0 software (Stata Corporation, College Station, Texas, USA). Pooled ORs with $95 \%$ CI will be calculated for dichotomous outcomes, mean differences (MDs) with $95 \%$ CI for continue outcomes. Heterogeneity of treatment effects across trials will be assessed by $c^{2}$ and $\mathrm{I}^{2}$ statistics. If the $\mathrm{p}$ value $\geq 0.1$ and $\mathrm{I}^{2}$ is $\leq 50 \%$, it suggests that there is no statistical heterogeneity, and the Mantel-Haenszel fixed effects model will be used for meta-analysis. If the $\mathrm{p}$ value $<0.1$ and $\mathrm{I}^{2}$ is $>50 \%$, we will explore sources of heterogeneity by subgroup analysis and meta-regression. If there is no clinical heterogeneity, the Mantel-Haenszel random effects model will be used to perform meta-analysis. ${ }^{23}$ Reporting bias will be examined using the Begg's and Egger's funnel plot method. ${ }^{25}{ }^{26}$ In addition, the contour-enhanced funnel plot will be used as an aid to distinguish asymmetry due to publication bias from that due to other factors. ${ }^{27}$

\section{Trial sequential analysis}

$\mathrm{TSA}^{18}$ will be performed to reduce the risk of random errors. We will add trials according to the year of publication, and if more than one trial is published in a year, the trials will be alphabetically added according to the last name of the first author. ${ }^{28}$ TSA will be performed for dichotomous outcomes as well as for continuous outcomes, to control the risks of random errors due to sparse data and multiplicity. ${ }^{29}{ }^{30}$ We will also adapt a relative risk reduction of $20 \%$, an $\alpha$ (type I error) of $5 \%$, a $\beta$ (type II error) of $20 \%$ and the diversity of the meta-analysis. ${ }^{19} 30$

\section{Geometry of the network}

A network plot will be drawn to describe and present the geometry of the treatment network of comparisons across trials to ensure if a network meta-analysis is feasible. Trials will be excluded if the trials are not connected by treatments. Network geometry will use nodes to represent different interventions and edges to represent the head-to-head comparisons between interventions. The size of nodes and thickness of edges are associated with sample sizes of intervention and numbers of included trials, respectively.

\section{Network meta-analysis}

A Bayesian network meta-analysis will be performed using WinBUGS 1.4.3 software (MRC Biostatistics Unit, Cambridge, UK). The random and fixed effect models with vague priors for multiarm trials developed by Ade $e t a l^{11}$ will be used. The pooled estimation and the probability of which treatment is the best will be obtained using the Markov Chains Monte Carlo method. Three Markov Chains will be run simultaneously with different arbitrarily chosen initial values. We will first generate 50000 simulations for each chain, and these simulations will then be discarded as the 'burn-in' period. Then posterior summaries will be based on 100000 subsequent simulations. The model convergence will be assessed by trace plots and Brooks-Gelman-Rubin plots. ${ }^{32}$ The statistical heterogeneity in the entire network will be assessed on the bias of the magnitude of heterogeneity variance parameter $\left(\mathrm{I}^{2}\right.$ or $\left.\tau^{2}\right)$ estimated from the network meta-analysis models using R-3.2.2 software (R Foundation for Statistical Computing, Vienna, Austria). The results of dichotomous outcomes will be reported as posterior medians of OR with $95 \%$ credible intervals (CrIs), and medians of MD with $95 \% \mathrm{CrI}$ for continue outcomes. If a loop connecting three arms exists, inconsistency between direct and indirect comparisons will be evaluated using a node splitting method. ${ }^{33}$ The choices between fixed and random effect models, consistency and inconsistency models, will be made by comparing the deviance information criteria (DIC) for each model. $^{34}{ }^{35}$ The model with the lowest DIC will be preferred (differences $>3$ are considered significant).

Clinical decisions about the choice of treatments can be recommended based on the probability results of ranking when the differences in effect size of different treatments are small. ${ }^{36}$ The surface under the cumulative ranking area (SUCRA) will be calculated to summarise and report the probability values. SUCRA values are expressed as percentages-SUCRA value will be $100 \%$ for the best treatment, while SUCRA value will be $0 \%$ for the worst treatment. ${ }^{37}$

In order to explore the sources of heterogeneity or inconsistency in the entire network, we will perform network meta-regression or subgroup analysis. Network meta-regression will be conducted using random effects network meta-regression models to examine potential effect moderators such as size of mesh, number of tack, follow-up and sample size.

If we include enough trials per comparison, a sensitivity analysis will be conducted. We will conduct a sensitivity analysis excluding trials that are missing relative data, and we will conduct another sensitivity analysis excluding trials with a total sample size of $<50$ randomised patients.

The quality of evidence will be classified by the GRADE group into four levels-high quality, moderate quality, low quality and very low quality. ${ }^{38}$ This process will be performed using GRADE pro 3.6 software (http://www.gradeworkinggroup.org/).

Furthermore, a comparison-adjusted funnel plot will be conducted to identify whether there will be a small sample effect among intervention networks, using STATA V.12.0 software (Stata Corporation, College Station, Texas, USA).

\section{ETHICS AND DISSEMINATION \\ Ethical issues}

Ethical approval and patient consent are not required since this is a meta-analysis based on published studies.

\section{Publication plan}

This protocol has been registered on the international prospective register of systematic reviews 
(PROSPERO). ${ }^{39}$ The procedures of network meta-analysis will be conducted according to the PRISMA extension statement for reporting of systematic reviews incorporating network meta-analyses of healthcare interventions. The results of this network meta-analysis and TSA will be submitted to a peerreviewed journal for publication.

\section{Author affiliations}

${ }^{1}$ The First Clinical Medical College of Lanzhou University, Lanzhou, China

${ }^{2}$ Evidence-based Medicine Center of Lanzhou University, Lanzhou, China

${ }^{3}$ Key Laboratory of Evidence-based Medicine and Knowledge Translation of Gansu Province, Lanzhou, China

${ }^{4}$ Department of Breast-Thyroid Surgery, The Second Xiangya Hospital of Central South University, Changsha, China

${ }^{5}$ Department of Gastrointestinal Surgery, Xijing Hospital of Digestive Diseases, Xijing Hospital, Four Military Medical University, Xi'an, China

Acknowledgements The authors are grateful to Mogo Editing for polishing and revising the language.

Contributors LG, J-hT and K-hY planned and designed the research; LL and WQ tested the feasibility of the study; LG wrote the manuscript; LG, J-hT and $\mathrm{K}-\mathrm{hY}$ approved the final version of the manuscript.

Competing interests None declared.

Provenance and peer review Not commissioned; externally peer reviewed.

Open Access This is an Open Access article distributed in accordance with the Creative Commons Attribution Non Commercial (CC BY-NC 4.0) license, which permits others to distribute, remix, adapt, build upon this work noncommercially, and license their derivative works on different terms, provided the original work is properly cited and the use is non-commercial. See: http:// creativecommons.org/licenses/by-nc/4.0/

\section{REFERENCES}

1. Rutkow IM, Robbins AW. Demographic, classificatory, and socioeconomic aspects of hernia repair in the United States. Surg Clin North Am 1993;73:413-26.

2. Rutkow IM. Epidemiologic, economic, and sociologic aspects of hernia surgery in the United States in the 1990s. Surg Clin North Am 1998;78:941-51, v-vi.

3. Burcharth J, Pedersen M, Bisgaard T, et al. Nationwide prevalence of groin hernia repair. PLoS ONE 2013;8:e54367.

4. Bisgaard $\mathrm{T}$, Kehlet $\mathrm{H}$, Bay-Nielsen $\mathrm{M}$, et al. A nationwide study on readmission, morbidity, and mortality after umbilical and epigastric hernia repair. Hernia 2011;15:541-6.

5. Treadwell J, Tipton K, Oyesanmi O, et al. Surgical options for inguinal hernia: comparative effectiveness review. Rockville, MD: Agency for Healthcare Research and Quality (US), 2012; Report No: 12-EHC091-EF

6. Colvin HS, Rao A, Cavali M, et al. Glue versus suture fixation of mesh during open repair of inguinal hernias: a systematic review and meta-analysis. World J Surg 2013;37:2282-92.

7. McCormack K, Scott NW, Go PM, et al. Laparoscopic techniques versus open techniques for inguinal hernia repair. Cochrane Database Syst Rev 2003;(1):CD001785.

8. Awad S, Fagan S. Current approaches to inguinal hernia repair. Am J Surg 2004;118:9S-16S.

9. Sanders DL, Waydia S. A systematic review of randomised control trials assessing mesh fixation in open inguinal hernia repair. Hernia 2014;18:165-7.

10. Ladwa N, Sajid MS, Sains $P$, et al. Suture mesh fixation versus glue mesh fixation in open inguinal hernia repair: a systematic review and meta-analysis. Int J Surg 2013;11:128-35.

11. Prieto $M$, Gastaca M, Agirre L, et al. The comparison of self-gripping mesh and sutured mesh in open inguinal hernia repair: the results of meta-analysis. Ann Surg 2015. Published Online First 10 Oct 2015. doi:10.1097/SLA.0000000000000989.

12. Fang Z, Zhou J, Ren F, et al. Self-gripping mesh versus sutured mesh in open inguinal hernia repair: system review and meta-analysis. Am J Surg 2014;207:773-81.
13. Pandanaboyana S, Mittapalli D, Rao A, et al. Meta-analysis of self-gripping mesh (Progrip) versus sutured mesh in open inguinal hernia repair. Surgeon 2014;12:87-93

14. $\mathrm{Li} \mathrm{J}$, Ji Z, Li Y. The comparison of self-gripping mesh and sutured mesh in open inguinal hernia repair: the results of meta-analysis. Ann Surg 2014;259:1080-5.

15. Zhang $\mathrm{C}$, Li F, Zhang $\mathrm{H}$, et al. Self-gripping versus sutured mesh for inguinal hernia repair: a systematic review and meta-analysis of current literature. J Surg Res 2013;185:653-60.

16. Bafeta A, Trinquart L, Seror R, et al. Reporting of results from network meta-analyses: methodological systematic review. BMJ 2014;348:g1741.

17. Thorlund K, Engstrom J, Wetterslev J, et al. User manual for trial sequential analysis (TSA). Copenhagen Trial Unit. http://www.ctudk/ tsa/files/tsa-manualpdf (accessed 2015).

18. Unit CT. TSA-trial sequential analysis. http://ctu.dk/tsa/ (accessed 3 Jul 2015).

19. Wetterslev J, Thorlund K, Brok J, et al. Estimating required information size by quantifying diversity in random-effects model meta-analyses. BMC Med Res Methodol 2009;9:86.

20. Thorlund $\mathrm{K}$, Imberger $\mathrm{G}$, Walsh $\mathrm{M}$, et al. The number of patients and events required to limit the risk of overestimation of intervention effects in meta-analysis — a simulation study. PLOS ONE 2011;6:e25491.

21. Moher D, Shamseer L, Clarke $M$, et al. Preferred reporting items for systematic review and meta-analysis protocols (PRISMA-P) 2015 statement. Syst Rev 2015;4:1.

22. Hutton B, Salanti G, Caldwell DM, et al. The PRISMA extension statement for reporting of systematic reviews incorporating network meta-analyses of health care interventions: checklist and explanations. Ann Intern Med 2015;162:777-84

23. Higgins JPT, Green S. Cochrance handbook for systematic reviews of interventions version 5.1.0[EB/OL]. The Cochrane Collaboration, 2011[2013.5.16]. http://www.cochrane-handbook.org

24. Gurusamy KS. Management strategies for pancreatic pseudocysts: a network meta-analysis. Cochrane Database Syst Rev 2014;11: CD011392.

25. Begg CB, Mazumdar M. Operating characteristics of a rank correlation test for publication bias. Biometrics 1994;50:1088-101.

26. Egger M, Davey Smith G, Schneider M, et al. Bias in meta-analysis detected by a simple, graphical test. BMJ 1997;315:629-34.

27. Peters JL, Sutton AJ, Jones DR, et al. Contour-enhanced meta-analysis funnel plots help distinguish publication bias from other causes of asymmetry. J Clin Epidemiol 2008;61:991-6.

28. Ganzevoort W, Alfirevic Z, von Dadelszen P, et al. STRIDER sildenafil therapy in dismal prognosis early-onset intrauterine growth restriction-a protocol for a systematic review with individual participant data and aggregate data meta-analysis and trial sequential analysis. Syst Rev 2014;3:23.

29. Brok J, Thorlund K, Gluud C, et al. Trial sequential analysis reveals insufficient information size and potentially false positive results in many meta-analyses. J Clin Epidemiol 2008;61:763-9.

30. Wang $\mathrm{Q}, \mathrm{He} X \mathrm{R}$, Tian $\mathrm{JH}$, et al. Pancreatic duct stents at pancreaticoduodenectomy: a meta-analysis. Dig Surg 2013;30:415-24.

31. Ades AE, Welton N, Lu G. Introduction to mixed treatment comparisons[EB/OL][2015.3.20]. http://www.bristol.ac.uk/ social-community-medicine/media/mpes/intro-to-mtc.pdf

32. Gelman A, Rubin DB. Inference from iterative simulation using multiple sequences. Stat Sci 1992;7:457-72.

33. Lu G, Ades AE. Combination of direct and indirect evidence in mixed treatment comparisons. Stat Med 2004;23:3105-24.

34. Dias S, Sutton AJ, Ades AE, et al. Evidence synthesis for decision making 2: a generalized linear modeling framework for pairwise and network meta-analysis of randomized controlled trials. Med Decis Making 2013;33:607-17.

35. Spiegelhalter DJ, Best NG, Carlin BP, et al. Bayesian measures of model complexity and fit. J Royal Statistical Soc (B) 2002;64:583-616.

36. Wu HY, Huang JW, Lin HJ, et al. Comparative effectiveness of renin-angiotensin system blockers and other antihypertensive drugs in patients with diabetes: systematic review and Bayesian network meta-analysis. BMJ 2013;347:f6008.

37. Salanti G, Ades AE, loannidis JP. Graphical methods and numerical summaries for presenting results from multiple-treatment meta-analysis: an overview and tutorial. J Clin Epidemiol 2011;64:163-71.

38. Puhan MA, Schünemann HJ, Murad MH, et al. A GRADE Working Group approach for rating the quality of treatment effect estimates from network meta-analysis. BMJ 2014;349:g5630.

39. Ge L, Tian JH, Li L, et al. Mesh fixation methods in open inguinal hernia repair: a protocol for network meta-analysis and trial sequential analysis of randomized controlled trials. PROSPERO 2015:CRD42015023758. http://www.crd.york.ac.uk/PROSPERO/ display_record.asp?ID=CRD42015023758 